\title{
Glottic Cancer pT1b TNM Finding v8
}

National Cancer Institute

\section{Source}

National Cancer Institute. Glottic Cancer pT 1b TNM Finding v8. NCI Thesaurus. Code C133113.

Glottic cancer with tumor involving both vocal cords. (from AJCC 8th Ed.) 